BULLETIN OF THE

AMERICAN MATHEMATICAL SOCIETY

Volume 80, Number 4, July 1974

\title{
METRIZATION AND PARACOMPACTNESS IN TERMS OF REAL FUNCTIONS
}

\author{
BY J. A. GUTHRIE AND MICHAEL HENRY
}

Communicated by Mary Ellen Rudin, November 15, 1973

One of the most useful tools developed over the last fifty years in the theory of metrization of topological spaces is the following: "A $T_{0}$ space $X$ is separable metrizable if and only if $X$ has the weak topology induced by countably many real-valued functions." This embedding theorem appears in various forms in most text books, and is customarily used in proving the Urysohn metrization theorem. Of course, the Urysohn metrization theorem is itself a corollary to the Nagata-Smirnov theorem.

It is the purpose of this paper to announce a recent result that will serve as an analogue of the above embedding theorem for arbitrary metric spaces, in that the embedding theorem follows as a corollary and the Nagata-Smirnov theorem follows as a consequence.

Call a collection $\left\{f_{\alpha}: \alpha \in A\right\}$ of real-valued functions defined on a topological space relatively complete if $\inf \left\{f_{\beta}: \beta \in B\right\}$ and $\sup \left\{f_{\beta}: \beta \in B\right\}$ are continuous functions for each $B \subset A$. Then it is true that

THEOREM 1. A $T_{0}$ space $X$ is metrizable if and only if $X$ has the weak topology induced by a $\sigma$-relatively complete collection.

The proof is accomplished by showing that $X$ is a paracompact Hausdorff space with a development. The paracompactness is an immediate consequence of the following result.

THEOREM 2. A $T_{1}$ space $X$ is paracompact if and only if for each open cover $\mathscr{W}$, there is a $\sigma$-relatively complete collection $\bigcup\left\{f_{n \alpha}: \alpha \in A_{n}\right\}$ and a refinement of $\mathscr{W}$ of sets of the form $\bigcap_{i=1}^{m} f_{n_{i} \alpha_{i}}^{-1}\left(U_{i}\right)$ where $U_{i}$ is open in $R$.

Department of Mathematics, University of Pittsburgh, Pittsburgh, PennsylVANIA 15213

Department of Mathematics, West Virginia University, Morgantown, West VIRGINIA 26506 $54 \mathrm{C} 30$.

AMS (MOS) subject classifications (1970). Primary 54E35, 54D20; Secondary Copyright @ American Mathematical Society 1974 